\title{
Efficient Method of Deriving Functional Endothelial Progenitor Cells From Hematopoietic Stem Cells
}

\author{
Shetty $\mathrm{P}^{*}$, Shah K and Viswanathan C \\ Regenerative Medicine Group, Reliance Life Sciences Pvt. Ltd., Rabale, Navi Mumbai, India
}

*Corresponding author: Shetty P, Regenerative Medicine Group, Reliance Life Sciences Pvt. Ltd., R-282, TTC Area of MIDC, Thane-Belapur Road, Rabale, Navi Mumbai, India, Fax: 091 2240678099, Tel: 091 2240678352,E-mail: prathibha.shetty@relbio.com

Citation: Shetty P, Shah K, Viswanathan C (2015) Efficient Method of Deriving Functional Endothelial Progenitor Cells From Hematopoietic Stem Cells. J Hematol Blood Disord 1(1): 102. doi: 10.15744/24557641.1.102

Received Date: January 21, 2015 Accepted Date: August 03, 2015 Published Date: August 04, 2015

\begin{abstract}
EPCs (Endothelial progenitor cells) are the precursors of endothelial cells that form the inner lining of blood vessels and are involved in neovascularization and re-endothelializaton of injured tissues. These cells have the potential for treating a multitude of angiogenic disorders giving a new hope in the arena of vascular biology. In the present study, we have demonstrated that cord blood derived expanded $\mathrm{CD} 34^{+}$cells can be differentiated into functional EPCs in vitro. Differentiation was performed using three different media conditions: media with a combination of various cytokines, media devoid of cytokines and a commercial medium. The differentiated cells were characterized based on their morphology as well as at the molecular and cellular level using RT-PCR, FACS and IC. Functionality of the differentiated cells was also analyzed by various assays such as dye uptake, lectin binding and in vitro tube formation. In brief, the results showed that EPCs cultured in the in-house media devoid of cytokines exhibited both CFU-ECs (Colony forming unit endothelial cells) and ECFCs (Endothelial colony forming cells). These differentiated cells expressed endothelial markers such as CD34, CD105, VEGFR2, VEGFR3, vWF, ecNOS and CD31 at a higher rate as compared to the other media studied, were also functional and secreted growth factors such as VEGF and bFGF. Thus, in this study, we have tried to derive an economically feasible and a scalable approach in successfully differentiating expanded CD $34^{+}$cells from cord blood to functional EPCs. However, the safety and efficacy of the differentiated cells needs to be determined in suitable preclinical animal models and further clinical studies.
\end{abstract}

Keywords: Human umbilical cord blood; Hematopoietic stem cells; Endothelial progenitor cells; Tube formation; Vascular cytokines

\section{Introduction}

EPCs as the name suggests are the progenitor cells for endothelial cells that line the blood vessels. They play a major physiological role in maintaining vascular integrity. They home to the ischemic and tumor tissues and aid in their revascularization. They have also been used as a biomarker or as a cell therapy in various clinical trials to treat patients with heart disease, diabetes, peripheral arterial disease, pulmonary disease and cancer despite the ambiguity in fully characterizing it. Thus, EPCs have many functions and the area of vascular biology involving endothelial cells is slowly emerging in treating various life-threatening pathologies [1].

EPCs stimulate angiogenesis or vascularization either by directly differentiating into mature endothelial cells or by acting via paracrine stimuli thereby stimulating the formation and repair of endothelium [2]. Vasculogenesis and Angiogenesis are two different processes of blood vessel formation with different types of cells involved in it. The former process of blood vessel formation occurs during embryogenesis from EPCs or angioblasts with blood island formation comprising of EPCs at the periphery and HSCs at the center [3]. These blood islands fuse resulting in the formation of primary vascular plexus composed of fine capillaries and this marks the end of vasculogenesis [3,4]. The latter process of blood vessel formation occurs in adult life where further transformations/ branching of the already formed vascular plexus takes place by proliferation, migration \& remodeling of preexisting mature endothelial cells (ECs). It occurs by 2 distinct methods:- Sprouting (SA) and non-sprouting angiogenesis or intussesceptive angiogenesis (IA) [3,5]. However, ample evidence showing endothelial cells derived from bone-marrow existing on the site of new blood vessel formation has changed the notion that vasculogenesis occurs only during embryogenesis thereby indicating the participation of vasculogenesis in postnatal neovascularization [6].

It was also shown by Asahara T et al. 1997 [7] that bone-marrow derived circulating EPCs are present in adult human peripheral blood which are supposedly derived from $\mathrm{CD}_{3} 4^{+}$mononuclear cells $\left(\mathrm{MNC}^{\mathrm{CD} 34+}\right)$. Moreover, these circulating EPCs not only differentiated into ECs in vitro but also incorporated into sites of neovascularization when transplated in vivo in mature immunodeficient animals. 
Though molecular events involved in the derivation and differentiation of endothelial cells (ECs) from the early mesoderm remain uncertain, but quite a few papers suggests that both HSCs and EPCs develop together from a common progenitor shortly after embryo implantation with the formation of blood islands within the primitive yolk sac. Due to the common origin of both HSCs and EPCs, there are many markers shared between both the hematopoietic and endothelial lineages like CD34, KDR, Tie-2/Tek. However, the major difference is endothelial cells are positive for CD34 but negative for CD45 and this distinguishes them from HSCs which are positive for both the markers [8].

HSCs (hematopoietic stem cells) or HPCs (hematopoietic progenitor cells) are denoted as CD34 ${ }^{+}$cells and EPCs, a group of circulating cells are thought to be derived from these cells [2]. Human bone marrow, umbilical cord blood and granulocyte colonystimulating factor (G-CSF)-mobilized peripheral blood have been used as sources to isolate CD34 ${ }^{+}$cells which contains EPCs [6]. Bone marrow is considered to be the most primitive source of HSCs but the process of obtaining bone marrow is painful. Also, the donor's age affects the differentiation ability of stem cells obtained from them. Peripheral blood contains fewer amounts of HSCs. So, umbilical cord blood serves as the best source since it is a non-controversial medical waste after child birth and large quantities of cells can be obtained from them through a painless procedure [8]. Cord-blood yields about 0.2-1\% EPCs as compared to 0.002 to $0.01 \%$ EPCs obtained from peripheral blood. Moreover, cord-blood derived EPC colonies not only emerged earlier but also appeared larger in size and contained smaller cells than adult colonies derived from bone marrow or peripheral blood. Also, endothelial cells derived from cord blood EPCs have extensive proliferative potential in vitro with atleast 100 population doublings and retain high levels of telomerase activity [9].

The most widely accepted method for phenotypic characterization of EPCs is the expression of markers such as CD34 \& VEGFR2 (KDR/ CD309/ Flk-1) along with their ability of taking up acetylated-LDL and binding ulex lectin. The ability to form colony like structures when cultured on fibronectin and tubule-like structures in matrigel describe the functional capacity of EPCs [2].

In this manuscript, we aim to derive functional EPCs from a readily available source (cord-blood CD34 ${ }^{+}$cells) using simple protocols with two different in-house media conditions and to compare them with the commercial medium, EGM2 (Endothelial growth medium 2). EPCs so generated were characterized at their molecular and cellular level using RT-PCR, FACS and IF and also they were functionally assayed in-vitro. ELISA was also performed to check the concentration of VEGF and bFGF secreted by the differentiated cells. Based on our research findings, EPCs derived using the in-house culture system devoid of any cytokines were efficient in terms of their phenotypic and functional abilities in comparison to the cells derived from the commercial medium. Hence, the results obtained in this study indicates an economically cheaper method of deriving functional EPCs from HSCs considering the future clinical applications.

\section{Methods}

\section{Isolation and expansion of $\mathrm{CD}_{3} 4^{+}$cells from human umbilical cord blood}

CD $34^{+}$cells were isolated from human umbilical cord blood which was obtained after proper consenting process and approvals from IC-SCR (Institutional Committee for Stem Cell Research). This blood was processed to obtain MNCs (mononuclear cells). MNCs so obtained were subjected to magnetic bead separation for the isolation of CD $34^{+}$cells. Further, CD34 ${ }^{+}$cells were expanded in expansion media as per the protocol of Tipnis S, and Viswanathan C, 2010 [10]. The expanded cells were characterized by flow cytometry for expression of CD34 and they were found to be positively expressing CD34>80\%.

\section{Cell culture}

For the initial standardization of experiments, three different types of media were used. Medial contains basal medium IMDM

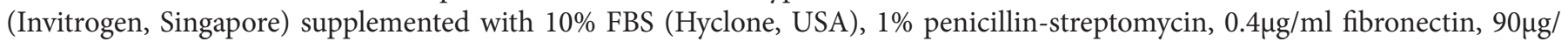
$\mathrm{ml}$ heparin (Loba Chemie, )and growth factors like 50ng/ml VEGF and 10ng/ml bFGF (R \& D Systems, USA). Media 2 includes IMDM supplemented with only $20 \% \mathrm{FBS}, 100 \mathrm{kU} / \mathrm{L}$ penicillin and $0.1 \mathrm{~g} / \mathrm{L}$ streptomycin. Media 3 is a commercially available medium known as endothelial growth medium-2 (EGM-2) containing 2\% FBS, hydrocortisone, hFGF-B, VEGF, R ${ }^{3}$-IGF-1, ascorbic acid, hEGF, gentamycin, amphotericin-B and heparin (Clonetics ${ }^{\mathrm{TM}}$ Lonza, Singapore) (Table 1). Expanded CD34 ${ }^{+}$cells were cultured at a density of $2 \times 10^{6}-3 \times 10^{6}$ cells in $75 \mathrm{~cm}^{2}$ flask in a humidified incubator at $37{ }^{\circ} \mathrm{C}, 5 \% \mathrm{CO}_{2}$ with all the types of media. After 48 hrs of culture, the non-adherent cells were transferred to a fresh flask. Fibronectin coating $(4 \mu \mathrm{g} / \mathrm{ml})$ was done for media type $2 \&$ 3 since they are devoid of fibronectin. Medium was replaced every 3-4 days. After 3-4 weeks, the cells were harvested and used for characterization studies. HUVEC $(<\mathrm{P}-5)$ cultured in EGM-2 was used as a positive control for all the experiments.

\begin{tabular}{|c|c|c|}
\hline NO & TYPE OF MEDIUM & MEDIA COMPOSITION \\
\hline 1 & Media $1(\mathrm{IMDM}+\mathrm{Fn})$ & $\begin{array}{l}\text { IMDM with } 10 \% \text { FBS, } 1 \% \text { Pen-Strep, } 0.4 \mu \mathrm{g} / \mathrm{ml} \text { fibronectin, } 90 \mu \mathrm{g} / \mathrm{ml} \text { heparin and } \\
\text { growth factors like } 50 \mathrm{ng} / \mathrm{ml} \text { VEGF and } 10 \mathrm{ng} / \mathrm{ml} \mathrm{bFGF}\end{array}$ \\
\hline 2 & Media 2 (IMDM) & IMDM with $20 \% \mathrm{FBS}, 100 \mathrm{kU} / \mathrm{L}$ penicillin and $0.1 \mathrm{~g} / \mathrm{L}$ streptomycin \\
\hline 3 & $\begin{array}{l}\text { Media } 3 \text { (EGM2 - } \\
\text { Endothelial Growth } \\
\quad \text { Medium 2) }\end{array}$ & $\begin{array}{c}\text { EBM2 (Endothelial basal medium 2) with growth supplements like } 2 \% \text { FBS, hydrocor- } \\
\text { tisone, hFGF-B, VEGF, R3-IGF-1, ascorbic acid, hEGF, gentamycin, amphotericin-B } \\
\text { and heparin }\end{array}$ \\
\hline
\end{tabular}

Table 1: Types of media used for derivation and differentiation of EPCs from CD $34^{+}$cells 


\section{Gene expression studies by Reverse Transcriptase PCR (RTPCR)}

Total RNA was extracted from pelleted cells $\left(\sim 0.5-1 \times 10^{6}\right)$ using an RNeasy ${ }^{\oplus}$ Kit (Qiagen, Singapore). First strand cDNA was synthesized from 1pg - $5 \mu \mathrm{g}$ of total RNA and Oligo $(\mathrm{dT})_{20}$ primers using Invitrogen's SuperScript III First Strand cDNA Synthesis kit (Invitrogen, Chromos Singapore) following the manufacturer's recommendations. RT-PCR was performed in a $12.5 \mu$ l reaction mixture with the appropriate forward and reverse primers, 2x PCR master mix (ABgene, Surrey, UK) and $1 \mu \mathrm{l}$ cDNA template. Cycling parameters were as follows: Initial denaturation at $94{ }^{\circ} \mathrm{C}$ for $45 \mathrm{sec}$ followed by 30 cycles of denaturation at $94{ }^{\circ} \mathrm{C}$ for $45 \mathrm{sec}$, annealing at $60{ }^{\circ} \mathrm{C}-70{ }^{\circ} \mathrm{C}$ for $45 \mathrm{sec}$ and extension at $72{ }^{\circ} \mathrm{C}$ for $1 \mathrm{~min}$ with a final extension at $72{ }^{\circ} \mathrm{C}$ for $10 \mathrm{mins}$. Primers were obtained from Sigma-Aldrich, India and they are listed in Table 2 with their corresponding annealing temperatures, along with their respective expected product size.

\begin{tabular}{|c|c|c|c|}
\hline $\begin{array}{l}\text { GENE } \\
\text { NAME }\end{array}$ & $\begin{array}{l}\text { FORWARD \& REVERSE } \\
\text { PRIMERS }\end{array}$ & $\begin{array}{l}\text { ANNEALING } \\
\text { TEMPERATURE }\end{array}$ & $\begin{array}{l}\text { EXPECTED PRODUCT } \\
\text { SIZE (bp) }\end{array}$ \\
\hline GAPDH & $\begin{array}{l}\text { 5'-TGAAGGTCGGAGTCAACGGAT-3' } \\
\text { 5'-CATGTGGGCCATGAGGTCCAC-3' }\end{array}$ & $60^{\circ} \mathrm{C}$ & 983 \\
\hline ecNOS & $\begin{array}{l}\text { 5'-AAGACATTTTCGGGCTCACGCTGCGCACCC-3' } \\
\text { 5'-TGGGGTAGGCACTTTAGTAGTTCTCCTAAC-3' }\end{array}$ & $60^{\circ} \mathrm{C}$ & 557 \\
\hline CD34 & $\begin{array}{l}\text { 5'-GTCTCACAGCCCTGCTTACC-3' } \\
\text { 5'-TGGGCGTAAGAGATGTCACC-3' }\end{array}$ & $60^{\circ} \mathrm{C}$ & 340 \\
\hline CD105 & $\begin{array}{l}\text { 5'-CACTAGCCAGGTCTCGAAGG-3' } \\
\text { 5'-CTGAGGACCAGAAGCACCTC-3' }\end{array}$ & $60^{\circ} \mathrm{C}$ & 165 \\
\hline $\begin{array}{l}\text { VEGFR2/ } \\
\text { KDR }\end{array}$ & $\begin{array}{l}\text { 5'-CAACAAAGTCGGGAGAGGAG-3' } \\
\text { 5'-ATGACGATGGACAAGTAGCC-3' }\end{array}$ & $64^{\circ} \mathrm{C}$ & 819 \\
\hline CD31 & $\begin{array}{l}\text { 5'-GCTGTTGGTGGAAGGAGTGC-3' } \\
\text { 5'-GAAGTTGGCTGGAGGTGCTC-3' }\end{array}$ & $62^{\circ} \mathrm{C}$ & 700 \\
\hline vWF & $\begin{array}{l}\text { 5'-AACCAGGGCGACGACTTCCTTA-3' } \\
\text { 5'-CTCGGAGAACCTGGTCATGCG-3' }\end{array}$ & $70^{\circ} \mathrm{C}$ & 156 \\
\hline
\end{tabular}

Table 2: List of primer sequences used for RT-PCR

\section{Immunophenotyping/ Fluorescence activated cell sorting (FACS)}

Immunophenotyping of the differentiated cells was done by flow cytometry. The adherent cells were washed with PBS and detached by incubating with $0.05 \%$ trypsin EDTA (Invitrogen, Chromos Singapore) for 5 minutes at $37^{\circ} \mathrm{C}$. After harvesting the adherent cells, cell count was taken and the cells were fixed using Cytofix/ Cytoperm (BD Pharmingen ${ }^{\mathrm{TM}}$ ) at $4{ }^{\circ} \mathrm{C}$ for 30 min. Approximately $0.05-0.1 \times 10^{6}$ cells per tube were used for cell surface antigen expression studies. The cells were incubated with CD31, CD34, CD45, HLA-DR, vWF (BD Biosciences Pharmingen, San Josè, CA, USA) and CD105, VEGFR2, VEGFR3 (R \& D Systems, Minneapolis, MN, USA). Appropriate isotype and secondary antibody controls from BD Biosciences Pharmingen were used during the assay. Goat anti-mouse FITC (BD Pharmingen, CA, USA) was used as a secondary antibody for vWF whereas IsoFITC (BD Pharmingen, CA, USA) was used for VEGFR2 and CD34, IsoPE (BD Pharmingen, CA, USA) for CD31, HLA-DR, CD105 and VEGFR3 antibodies and IsoPerCP (BD Pharmingen, CA, USA) for CD45. The cells were incubated with the respective antibodies at $4{ }^{\circ} \mathrm{C}$ for 30 mins. The pellet obtained after washing was resuspended in PBS and these cells were acquired on a FACS Calibur flow cytometer (Beckton Dickinson, USA) equipped with a $488 \mathrm{~nm}$ Argon Laser. Approximately 5000-10,000 events were acquired and analyzed using the Cell Quest software.

\section{Immunocytochemistry (IC)}

Differentiated cells harvested from the flasks were cultured in 8-well chamber slides and subjected to immunocytochemistry to analyze the expression of CD31, VEGFR2, vWF, CD105 and CD73. The adherent cells were washed twice with PBS and fixed with $4 \%$ paraformaldehyde (Sigma Aldrich) at $4{ }^{\circ} \mathrm{C}$ for 30 mins. Fixed specimens were washed once with PBS and incubated with primary antibodies of appropriate dilutions $(1: 100)$ at $4{ }^{\circ} \mathrm{C}$ overnight. After $24 \mathrm{hrs}$ incubation, the cells were washed twice with PBS and incubated at $4^{\circ} \mathrm{C}$ for $2 \mathrm{hrs}$ with secondary antibodies of appropriate dilutions (1:500). The cells were again washed twice with PBS and DAPI (4',6-diamidino-2-phenylindole) was added to each well and incubated for 2 mins on shaker. After the final washing step with PBS, the slides were mounted, dried overnight at $4{ }^{\circ} \mathrm{C}$ and then observed under a Zeiss fluorescent microscope.

\begin{tabular}{|c|c|}
\hline ANTIBODIES & BRAND \\
\hline $\begin{array}{c}\text { Primary antibodies } \\
\text { (CD73, CD31, CD34, CD45, HLA-DR, vWF), IsoPE/PerCP/FITC } \\
\text { (CD105, VEGFR2, VEGFR3) }\end{array}$ & $\begin{array}{l}\text { BD Pharmingen }{ }^{\mathrm{TM}} \\
\text { R \& D Systems }\end{array}$ \\
\hline $\begin{array}{l}\text { Secondary antibody (GAM FITC) } \\
\text { Goat antimouse antibody labeled with Alexa Fluor } 488\end{array}$ & $\begin{array}{l}\text { BD Pharmingen } \\
\text { Molecular Probes }^{\mathrm{TM}}\end{array}$ \\
\hline
\end{tabular}

Table 3: List of primary and secondary antibodies used in FACS and IC

\section{Cellular uptake of DiI (1,1'-dioctadecyl-3,3,3'3'-tetramethylindocarbocyanine perchlorate) labeled acetylated LDL (Low density lipoprotein) and binding of FITC labeled UEA-1 (Ulex europaues agglu- tinin-1)}


The differentiated cells were cultured in a $60 \mathrm{~mm}$ petri-dish at a density of $0.1 \times 10^{6}$ for $2-3$ days till they reached $80-90 \%$ confluency and then incubated with the respective medium containing $10 \mu \mathrm{g} / \mathrm{ml}$ DiI-Ac-LDL (Invitrogen, USA) for 4 hrs at $37^{\circ} \mathrm{C}$ in dark. Thereafter, the cells were washed with PBS and incubated with $10 \mu \mathrm{g} / \mathrm{ml}$ FITC-conjugated ulex lectin (Sigma-Aldrich, St.Louis, USA) for $1 \mathrm{hr}$ at $37^{\circ} \mathrm{C}$ in dark followed by washing and fixing them with $4 \%$ paraformaldehyde at $4{ }^{\circ} \mathrm{C}$ for 30 mins. The washing step was again repeated followed by staining of the cells with DAPI. After 2 final washing steps with $\mathrm{PBS}$ at $37^{\circ} \mathrm{C}$, the cells were analyzed for the uptake of DiI-Ac-LDL and binding of FITC-UEA-1 lectin using a Zeiss fluorescent microscope.

\section{Endothelial tube formation assay}

In-vitro functional capacity of EPCs to support endothelial tube formation was assessed in a co-culture system with HUVEC (P3P5) using BD Matrigel Matrix (BD Biosciences, Cat no: 354234). Ice cold Matrigel (50 $\mu \mathrm{l}$ ) was used for coating wells of a 96-well plate followed by incubation at $37^{\circ} \mathrm{C}$ for $1-2$ hours. Thereafter, $150 \mu \mathrm{l}$ EGM-2 medium containing 25,000 HUVECs and 150 $\mu \mathrm{l}$ EGM-2 medium containing 25,000 EPCs were added to the Matrigel and the cells were incubated for 6 hours at $37^{\circ} \mathrm{C}$ with $5 \% \mathrm{CO}$. EPCs used for the assay were labeled with DiI prior to seeding. DiI labeled Human fetal fibroblasts (HFFs) (P14) were used as a negative control for tube assay and were co-cultured with HUVEC similar to EPCs. Tube formation was assessed with an Olympus CKX41 inverted microscope using "Image J" software.

\section{VEGF and bFGF ELISA}

Cell culture supernatants of differentiated cells (EPCs) from $\mathrm{CD} 34^{+}$cells as well as HUVEC were collected and stored at $-20{ }^{\circ} \mathrm{C}$ till assayed. The concentration of VEGF and bFGF in the supernatant was determined using Quantikine VEGF and bFGF ELISA kit (R\&D Systems, Minneapolis, MN, USA) as per the manufacturer's instructions.

\section{Results}

\section{Morphological characteristics of $\mathrm{CD}^{+} 4^{+}$cells cultured and differentiated in-vitro}

Expanded and characterized $\mathrm{CD}_{3} 4^{+}$cells from human umbilical cord blood show a non-adhesive homogenous morphology (Figure 1A). These cells were initially cultured in tissue culture flasks for derivation of EPCs. After 48 hrs of incubation at $37{ }^{\circ} \mathrm{C}$ and $5 \% \mathrm{CO}_{2}$, non-adherent cells were transferred to a fresh flask in media 1 and fibronectin coated flasks in case of media 2 and 3. It was found that in media 1 and media 2, cells in the original flasks were elongated and spindle-shaped as compared to cells in the transfer flasks showing a heterogenous morphology with both round cells at the centre and spindle-shaped cells at the periphery referred to as CFU-EC (Figure 1B and 1C). In addition to this, ECFCs were also observed as shown in Figure 1E and 1F. It consisted of a cluster of round and spindle shaped cells evenly distributed throughout the colony. Cells cultured in the commercial medium EGM-2 showed a different pattern of morphology as compared to the other two media in which only ECFCs were detected with no CFU-ECs (Figure 1D). The differentiated cells were then characterized at the molecular and cellular level.
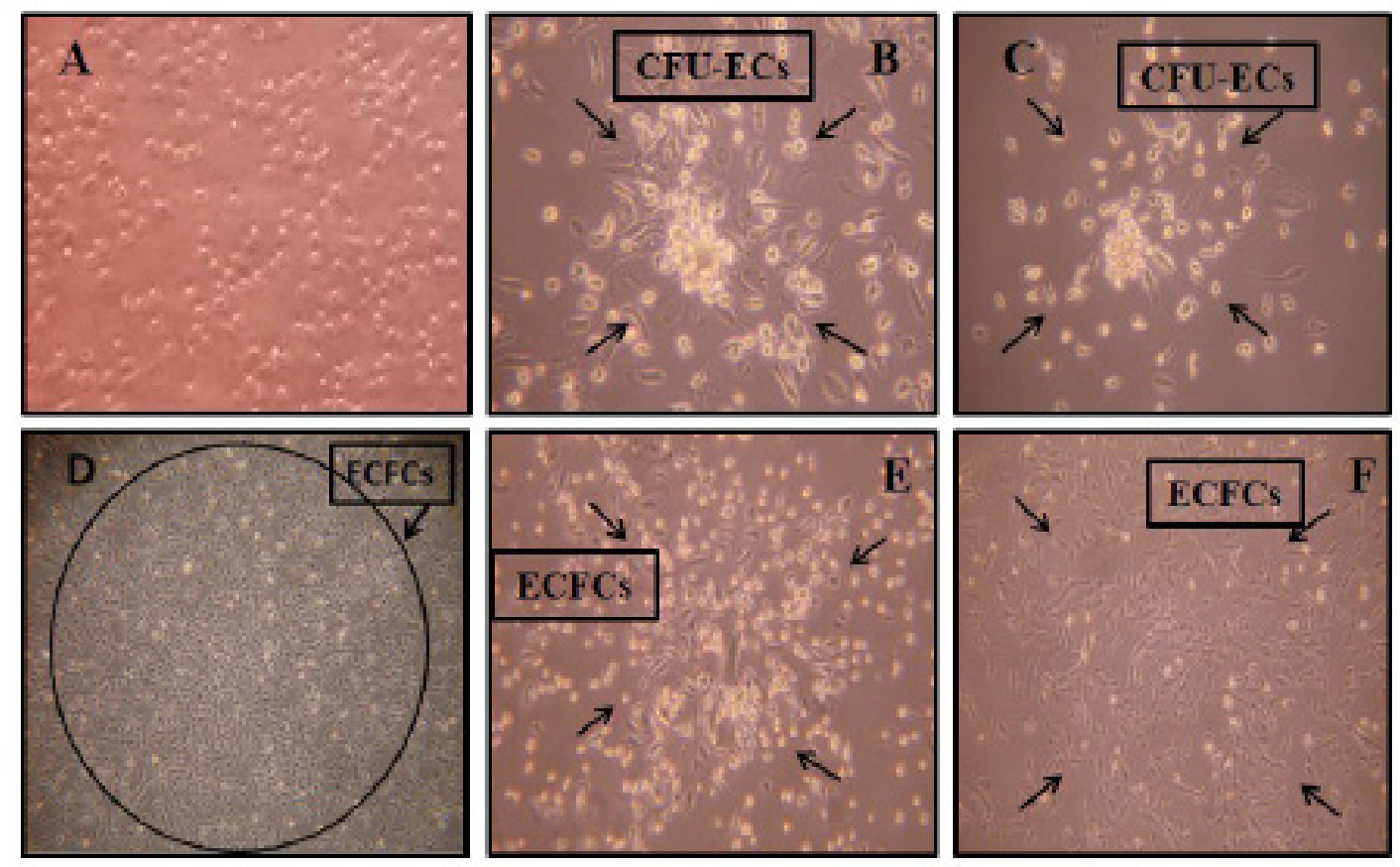

Figure 1: Morphology of undifferentiated and differentiated cells in the respective media:

A- Undifferentiated CD34 cells; B, E- EPCs in Media 1 (IMDM + Fn); C, F- EPCs in Media 2 (IMDM); D- EPCs in Media 3 (EGM2). Undifferentiated CD34 cells grow as suspension cultures. Both CFU-ECs and ECFCs were detected in differentiated cells when cultured in Media 1 and Media 2. Only ECFCs were detected in differentiated cells when cultured in Media 3. Both these colonies are considered to be proliferative EPC subtypes. The pictures of cells were taken at both $4 \mathrm{X}$ and 10X magnification. The arrows in the pictures indicate the colonies 


\section{Characterization of differentiated cells by RT-PCR}

Once the differentiated cells attained $70-80 \%$ confluency in the respective media, they were harvested and used for molecular analysis. Cell pellets were used for RNA isolation followed by conversion to cDNA. The cDNA was analyzed for markers expressed by endothelial cells such as ecNOS, CD105, CD34, CD31, KDR and vWF by RT-PCR. GAPDH was used as the house-keeping gene. All the genes were found to be expressed in differentiated cells (EPCs) and this was compared with HUVEC as a positive control. However, expanded CD34+ cells did not express CD105 and KDR but instead showed bands for ecNOS, CD34, CD31 and vWF as shown in Figure 2.

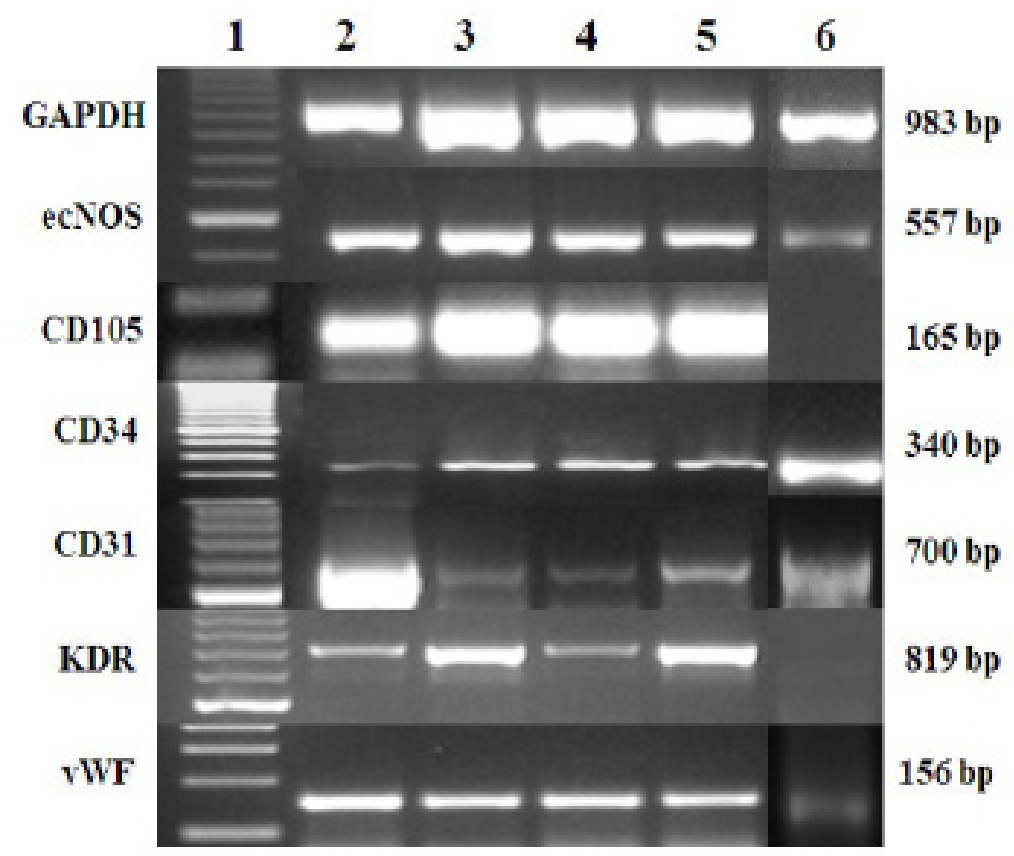

Figure 2: RT-PCR analysis of HUVEC, EPCs cultured in all the three media and CD34+ cells: Upon reaching $70-80 \%$ confluency, HUVEC, EPCs cultured in all the media and $\mathrm{CD} 34^{+}$cells were harvested and characterized by RTPCR for expression of a range of markers such as GAPDH, ecNOS, CD105, CD34, CD31, KDR and vWF. Lane 1 - 100bp ladder, Lane 2 - HUVEC, Lane 3 - EPCs (Media 1), Lane 4 - EPCs (Media 2), Lane 5 - EPCs (Media 3), Lane 6 - Expanded CD34+ cells

\section{Immunophenotypic characterization of differentiated cells at the cellular level using FACS and IC}

For cellular characterization, EPCs cultured in the respective media conditions were analyzed by FACS for expression of markers like CD105, CD31, vWF, VEGFR2, VEGFR3, HLA-DR, CD34 \& CD45 and compared with HUVEC as shown in Figure 3. Expression of CD105 and HLA-DR by EPCs cultured in media 2 was similar to HUVEC whereas expression of CD31, vWF and VEGFR2 in these differentiated cells was low. However, EPCs in this medium showed 62.26\% expression of VEGFR3 as compared to 51.75\% of HUVEC. Moreover, expression of CD34 and CD45 in these cells as well as HUVEC was negligible. Expanded CD34 cells were also stained for the above mentioned markers where they widely expressed all of them except VEGFR2 and VEGFR3. Thus, there was a down-regulation of CD34, CD45, CD31 and HLA-DR whereas up-regulation of CD105, vWF, VEGFR2 and VEGFR3 in EPCs cultured in media 2 as compared to expanded CD34+ cells. Overall, in-house media devoid of cytokines was better in terms of marker expression by EPCs as compared to cytokine-enriched media. EPCs cultured in different media conditions were also positively immunostained by IC for expression of endothelial markers like CD31, VEGFR2, vWF, CD105 and CD73 as shown in Figure 4 .

\section{Functional assays}

Cellular uptake of DiI-Ac-LDL and binding of FITC-UEA-1 lectin: EPCs derived from different media conditions were analyzed for their in vitro functionality by various assays such as uptake of DiI labeled acetylated LDL and binding of FITC labeled UEA-1 lectin. It was found that upon incubation with these compounds, HUVEC as well as EPCs cultured in all the media took up DiIAc-LDL and also bound FITC-UEA-1 lectin as shown by red and green staining respectively (Figure 5).

In vitro tubule formation: To assess the angiogenic potential of EPCs to form tubes in vitro, cells cultured in the respective media conditions were subjected to endothelial tube formation assay. In this assay, EPCs were labeled with DiI, a fluorescent dye (red) and cocultured with HUVEC (1:1 ratio) on a basement membrane matrix gel for 6 hours. It was observed that EPCs stimulated tube formation when co-cultured with HUVEC and the formation of tubes on matrigel was assessed by a Zeiss fluorescent microscope. Moreover, EPCs cultured in the respective media were traced to incorporate and integrate in both the branching points and lengths of tubules as shown by black arrows in Figures 6B-6D. In contrast, HFF inhibited tube formation by HUVEC and also formed clumps rather than incorporating in the tubules thus serving as a negative control for tube assay (Figure 6E). 


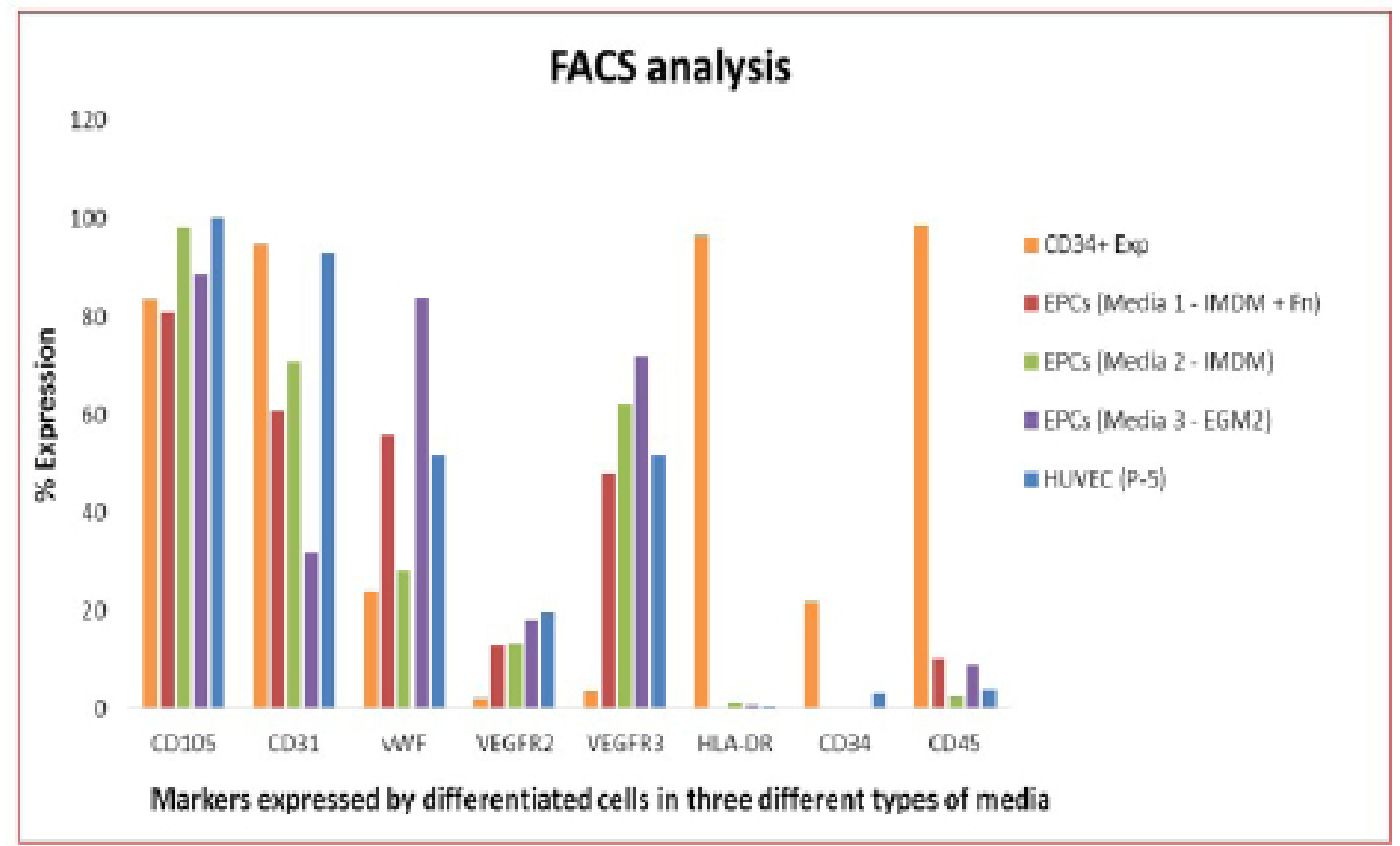

Figure 3: Bar graph showing a comparative overview of flow cytometric analysis of HUVEC, EPCs and CD34 ${ }^{+}$cells: Few markers such as CD105, CD31, vWF, VEGFR2, VEGFR3, HLA-DR, CD34 and CD45 were checked at the protein level by flow cytometry in HUVEC, EPCs cultured in all the media and expanded CD $34^{+}$cells. Expression of CD34, CD45, CD31 and HLA-DR got down-regulated whereas that of CD105, vWF, VEGFR2 and VEGFR3 got up-regulated in EPCs cultured in media 2 as compared to expanded CD34 $4^{+}$cells

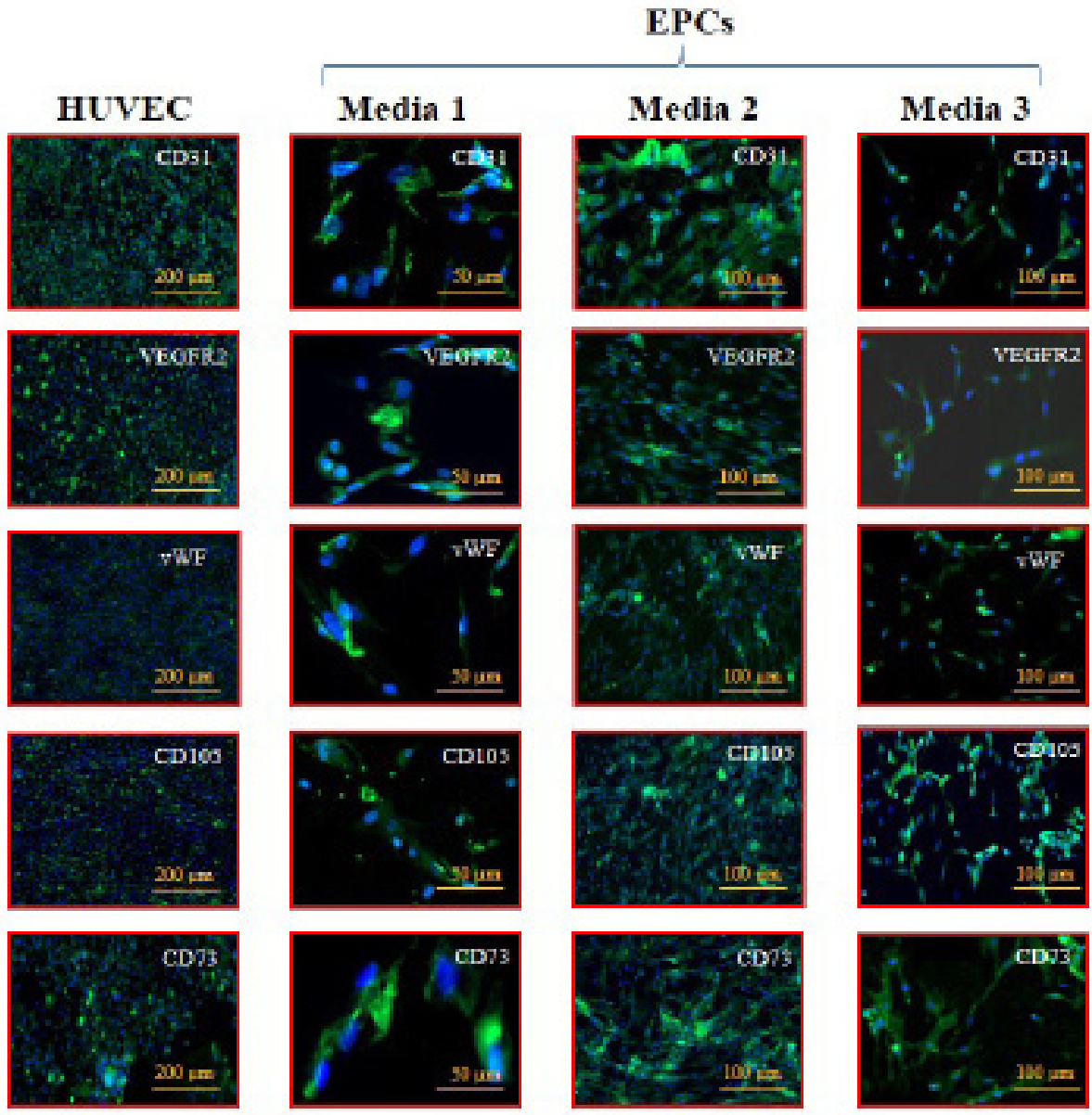

Figure 4: Staining of HUVEC and EPCs for immunocytochemistry: HUVEC and EPCs cultured in the respective media were immunostained for markers like CD31, VEGFR2, vWF, CD105 and CD73. All the markers were unlabeled except VEGFR2 which was labeled to FITC. Hence, for the detection of unlabeled antibodies, secondary antibody labeled to Alexa Flour 488 was used. The nucleus was stained blue by DAPI. The slides were observed at 5X, 10X and 20X magnifications using a Zeiss fluorescent microscope and accordingly the scale bars are provided 

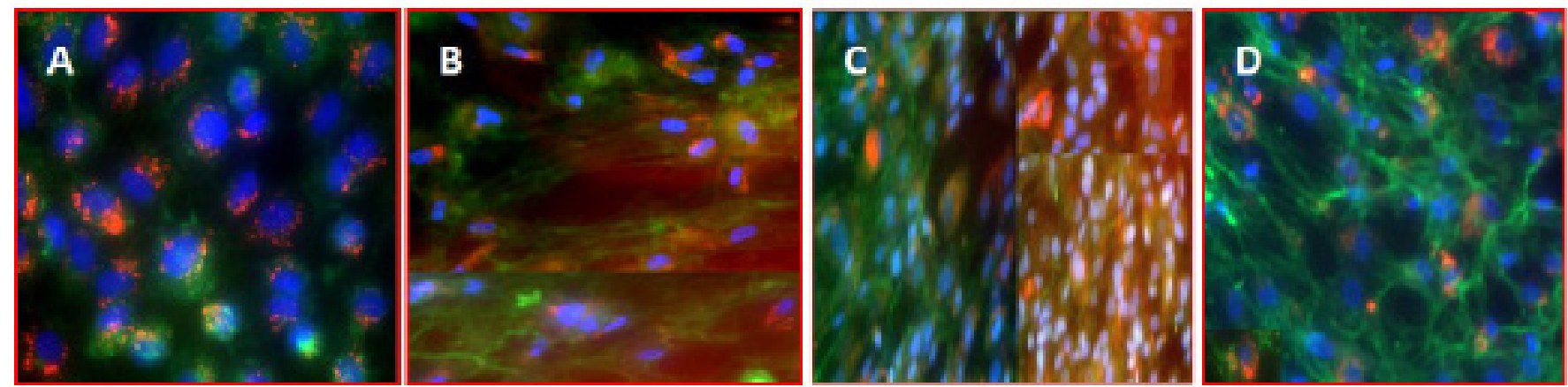

Figure 5: Uptake of DiI-Ac-LDL (red) and binding of FITC-UEA-1 lectin (green) by HUVEC and EPCs: A- HUVEC; B- EPCs cultured in Media 1; C- EPCs cultured in Media 2; D- EPCs cultured in Media 3. The nucleus was stained blue by DAPI. The culture dishes were observed at 10X and 20X magnification using a Zeiss fluorescent microscope. Various pictures were taken in different fields and the best pictures were cropped and clubbed together as seen as panels in Figures B and C. EPCs cultured in the respective media showed both red and green fluorescence indicating that they have taken up DiI-Ac-LDL and also bound FITC labeled UEA-1 lectin respectively which proves a part of their functionality in vitro and this has been compared with HUVEC
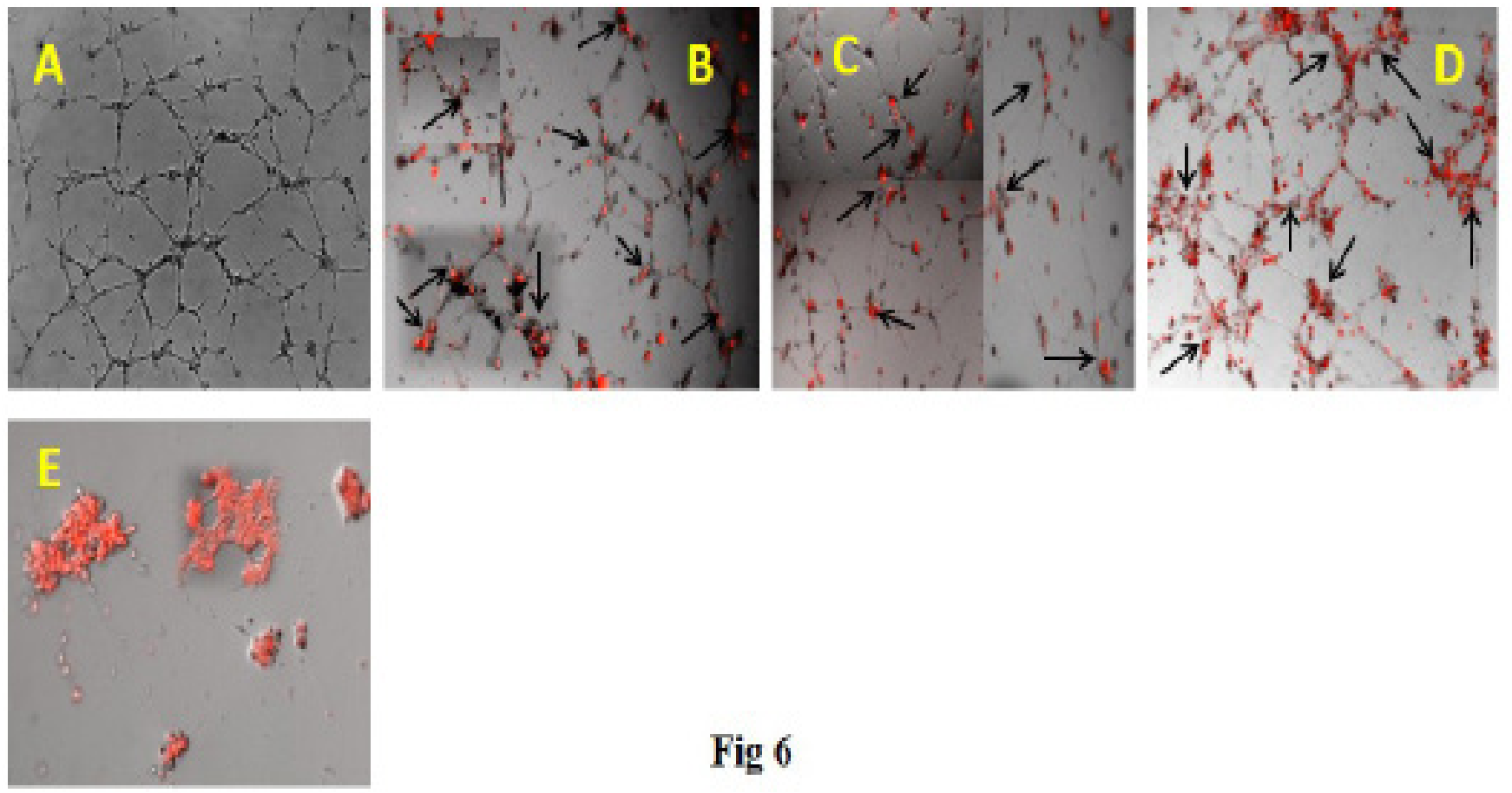

Figure 6: Capillary network formation by HUVEC alone and HUVEC co-cultured with EPCs \& HFF in a tube formation assay: A- HUVEC; B- HUVEC + EPCs (Media 1); C- HUVEC + EPCs (Media 2); D- HUVEC + EPCs (Media 3); E- HUVEC + HFF. EPC pre-stained with DiI (red) is traced along the tubuli and along the branching area of network formation (black arrows) [Figures B, C and D]. HFF pre-stained with DiI formed clumps and inhibited tube formation by HUVEC [Figure E]. Various pictures were taken at 5X magnification and the best pictures were cropped and clubbed together as seen as insets and panels in Figures B, C and E

\section{Cytokine analysis for secretion of VEGF and bFGF by EPCs}

It is reported that during the differentiation process, EPCs secrete a range of growth factors including VEGF and bFGF. The supernatant of EPCs cultured in all the media were analyzed for the presence of these cytokines by ELISA as shown in Figures 7A and 7B respectively. It was observed that cells cultured in the in-house media which contained high amounts of both VEGF and bFGF utilized these growth factors rather than secreting it in the medium as shown by their decreased concentration. Whereas, when EPCs were cultured in in-house media devoid of any growth factors, both VEGF and bFGF were secreted in the cell culture supernatant. Thus, the culture supernatant of differentiated cells in media 2 contained high VEGF concentration $(46 \mathrm{pg} / \mathrm{ml})$ and high bFGF concentration ( $10 \mathrm{pg} / \mathrm{ml}$ ) as compared to media alone. EGM2 is a commercial medium which consists of VEGF, bFGF and a cocktail of other growth factors. HUVEC was cultured in EGM2 which served as a positive control for endothelial cells. Hence, the secretion of VEGF and bFGF by HUVEC in presence of EGM2 was compared with EPCs cultured in the respective media. It was found that EPCs as well as HUVEC utilized VEGF and bFGF available to them rather than secreting it in EGM2 as shown by a profound decrease in concentration of both the growth factors. 


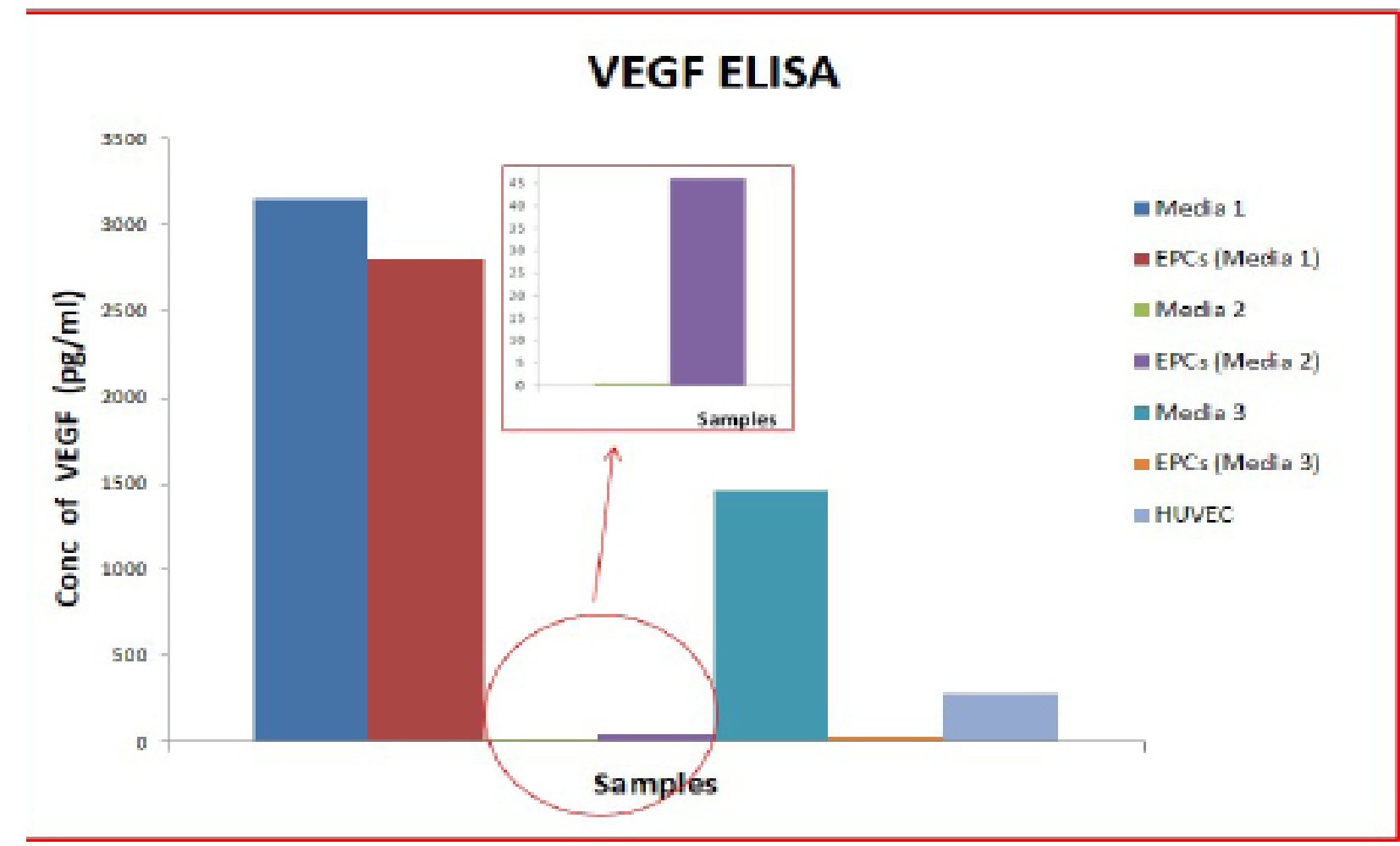

Figure 7A: ELISA for secretion of VEGF (pg/ml) by HUVEC and EPCs cultured in all the media: Secretion of VEGF in the culture supernatants of HUVEC and EPCs was checked by ELISA. EPCs cultured in media 2 secreted high concentration of VEGF (46 pg/ml) as compared to the other two media

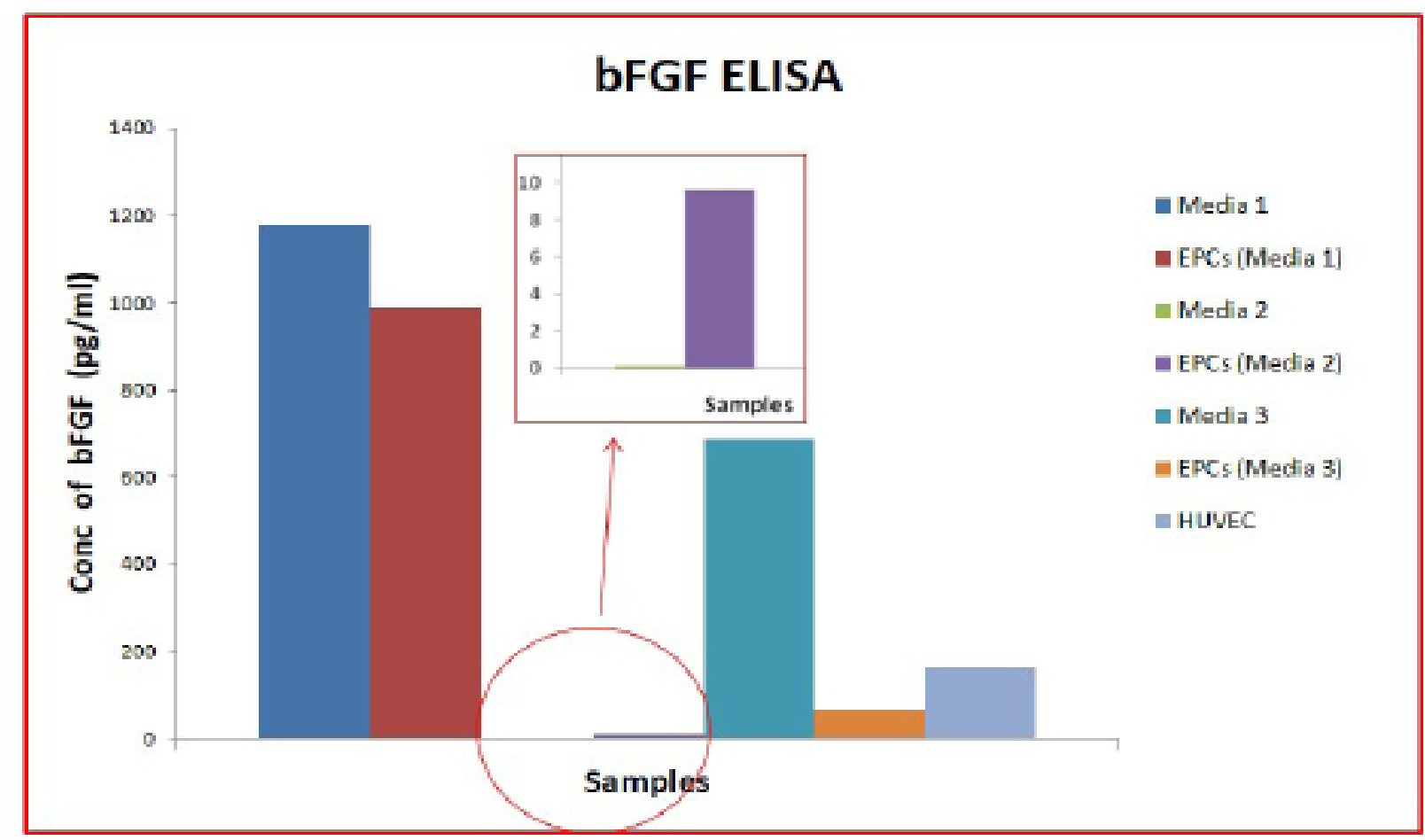

Figure 7B: ELISA for secretion of bFGF (pg/ml) by HUVEC and EPCs cultured in all the media: Secretion of bFGF in the culture supernatants of HUVEC and EPCs was checked by ELISA. EPCs cultured in media 2 secreted high concentration of bFGF (10pg/ml) as compared to the other two media 


\section{Discussion}

EPCs are a heterogeneous population of cells that display different phenotypes and functions according to the source, isolation, culture and characterization techniques employed [2]. There is no clear consensus till date as to when a circulating EPC differentiates into mature endothelial cell in vivo. However, it has been proposed that this differentiation may be initiated when EPCs migrate from the bone marrow into peripheral circulation and get terminated after their adhesion and insertion into the endothelial monolayer [11]. Thus, the differentiation process may be initiated when the progenitor cells are on their way to the injured tissue thereby increasing the available cell population capable of re-endothelialization of denuded arteries as well as neovascularization of ischemic regions [12]. Angiogenesis or vascularization can be stimulated by EPCs in two ways where they can either directly differentiate into mature endothelial cells or they can act via paracrine stimuli releasing various growth factors thus stimulating the formation and repair of endothelium [2]. One area where EPCs have been widely used as a cell therapy is in treating cardiovascular disorders. However, clinical studies pertaining to heart diseases have majorly utilized $1.5-125 \times 10^{6}$ BM MNCs (bone marrowmononuclear cells) by delivering them through intracoronary infusion [13].

In this study, cord-blood derived expanded CD $34^{+}$HPCs were aimed to differentiate into EPCs followed by their characterization and functionality testing in vitro. In view of this, three different types of media were compared to check for efficient differentiation. One of the key steps that led to the initial discovery of EPCs was using fibronectin-coated plates for culturing peripheral blood derived mononuclear cells [7]. Since then, EPCs have been generated from non-selected mononuclear cells or CD34 ${ }^{+}$and CD133 hematopoietic progenitors using fibronectin [2]. Also, the differentiation process of EPCs was accelerated when fibronectin was used as an ECM (extra cellular matrix). This was proved by Chun-Ling F et al. 2003 [6] who characterized in vitro differentiation of EPCs with gradual appearance of endothelial cell markers like Flk-1 and vWF and disappearance of stem cell marker AC133 when cord blood CD $34^{+}$cells were cultured on fibronectin for 7 days. Hence, the role of ECM such as fibronectin was assessed in media devoid of cytokines and as well as in the commerical medium. Upon differentiation, two types of colonies were observed, CFUECs and ECFCs in both the in-house media (Figure 1B,1C,1E \& 1F) whereas in presence of EGM2, cells formed only ECFCs and no CFU-ECs (Figure 1D). Thus, through this experiment, it has been detected for the first time in the field of EPC research that 2 different types of colonies emerged upon subjecting the same treatment. Case J et al. 2007 [8] describes CFU-ECs as hematopoietic lineage cells since they express CD45 whereas ECFCs gives rise to endothelial progeny as they are negative for CD45 expression. However, both the types of colonies are considered to be proliferative EPC subtypes which gives rise to functionally divergent EC progeny either in vitro or in vivo [14].

One of the major obstacles which limits detailed functional analysis and molecular characterization of EPCs is the relative scarcity of circulating cell population in vivo that displays the ability to trigger endothelial repair and angiogenesis [2]. Thus, an attempt has been made by us in this study to obtain EPCs from CD $34^{+}$cells in sufficient numbers and provide a gene expression profile of the differentiated cells, characterize them at their cellular (protein) level as well as perform functional assays.

Based on the phenotypic and functional characterization, we found that the in-house media devoid of cytokines proved to be best for derivation of EPCs from CD34+ cells as compared to the other media analyzed. A range of genes were checked for HUVEC, EPCs cultured in the respective media and expanded CD34 ${ }^{+}$cells such as ecNOS, CD105, CD34, CD31, KDR and vWF (Figure 2). Endothelial related markers were also checked at the protein level by flow cytometry such as CD105, CD31, vWF, VEGFR2, VEGFR3, HLA-DR, CD34 and CD45 as shown in Figure 3. Endothelial constitutive nitric oxide synthase (ecNOS) as the name suggests, is transcribed continuously and hence, was observed in all the differentiated cells as well as in CD34 cells albeit at a low intensity (Figure 2). CD31 was strongly expressed by both CD34 ${ }^{+}$cells and HUVEC but later got down-regulated in the differentiated cells at both the genetic and protein level. On the other hand, CD105 showed negligible expression in CD34 ${ }^{+}$cells but got up-regulated once the cells differentiated to EPCs and this was similar to HUVEC as shown in Figure 2. However, at the protein level, expanded CD $34^{+}$cells showed $83.57 \%$ expression of CD105 as compared to $98.22 \%$ expression by EPCs cultured in media 2 and $99.96 \%$ by HUVEC. Moreover, expression of vWF and VEGFR2 was low in CD34 $4^{+}$cells in contrast to its high expression by HUVEC and EPCs cultured in different media at both the genetic and protein level. Also, EPCs cultured in media 2 showed $62.26 \%$ expression of VEGFR3 as compared to $51.75 \%$ of HUVEC and $3.72 \%$ of CD $34^{+}$cells as shown from Figure 3 . On the contrary, HLA-DR was highly expressed at the protein level by CD $34^{+}$cells but its expression declined drastically in both HUVEC as well as the differentiated cells. Expression of CD34 was also intense in CD34 ${ }^{+}$cells but reduced in the differentiated cells as well as in HUVEC at both the genetic and protein level. A hematopoietic marker CD45 was also introduced which was strongly expressed by CD $34^{+}$cells (98.8\%) whereas there was a sudden drop in its expression by EPCs cultured in media 2 (2.22) which coincided with that of HUVEC (4.04). Thus, expression of CD34, CD45, HLA-DR and CD31 got down-regulated in EPCs cultured in media 2 as compared to $\mathrm{CD}_{3} 4^{+}$cells whereas ecNOS, CD105, vWF, VEGFR2 and VEGFR3 expression increased which was comparable to HUVEC. Thus, irrespective of the type of colony observed, cells differentiated from our experiments are believed to be endothelial progenitor cells (EPCs) and not hematopoietic cells as CD45 expression was found to be drastically reduced in them as compared to cord-blood derived expanded CD $34^{+}$cells. Moreover, immunocytochemistry of EPCs differentiated in the respective media was also done for the expression of markers like CD31, VEGFR2, vWF, CD105 and CD73 and all were found to stain positive (Figure 4). 
Apart from the phenotypic characterization by FACS and IC for expression of endothelial markers, the differentiated cells (EPCs) cultured in all the media showed positive uptake of DiI-Ac-LDL as well as bound FITC-UEA-1 lectin (Figure 5). Based on these functional assays, differentiated cells were confirmed to be EPCs as numerous research works have described EPCs based on this dye uptake method alone or in conjunction with FACS [2]. These dyes metabolically label endothelial cells thereby facilitating identification of cells of the endothelial lineage by fluorescence microscopy [15]. Functionally, endothelial cells are capable of forming tube-like structures in vitro when cultured on 3D culture systems composed of collagen or fibrin. The co-culture assay with HUVEC resulted in the incorporation and integration of EPCs within the branching points and lengths of endothelial tubules which was comparable to cells derived from the commercial media. This property of tube formation by endothelial cells on coculturing with HUVEC was affirmed by using a negative control, HFF. These cells not only inhibited tube formation by HUVEC but also clumped together rather than incorporating and integrating within the tubules (Figure 6).

Urbich and Dimmeler, 2004 [16] have reported that a marked secretion of growth factors like VEGF, HGF and IGF-1 were shown by EPCs when they were cultured from different sources. VEGF (Vascular Endothelial Growth Factor) is highly specific for cells of the endothelial lineage and hence the expression of this growth factor in EPCs was checked by ELISA. Secretion of another growth factor, bFGF (basic Fibroblast Growth Factor) was also checked. It was found that since media 1 and media 3 already contained VEGF and bFGF, the cells did not produce any more of these growth factors. Rather, the cells seemed to utilize these available growth factors as shown by their decreased concentration. Media 2 was devoid of any cytokines. So, the cells differentiated in this media themselves secreted $46 \mathrm{pg} / \mathrm{ml} \mathrm{VEGF}$ and $10 \mathrm{pg} / \mathrm{ml} \mathrm{bFGF} \mathrm{(Figure} \mathrm{7A} \mathrm{and} \mathrm{7B).} \mathrm{However,} \mathrm{the} \mathrm{release} \mathrm{of} \mathrm{various} \mathrm{other} \mathrm{growth}$ factors along with VEGF and bFGF may stimulate the proliferation, migration and survival of mature endothelial cells thereby influencing the classical process of angiogenesis [17].

Most of the research work in the literature involving endothelial cells has been done with EGM2 which is costly since it is a commercial media. Thus, an effort has been made in this study to provide a comparative analysis of different media conditions used to derive functional EPCs from cord-blood CD34 ${ }^{+}$cells thereby suggesting a cheaper alternative. Based on the results obtained, we found our simple in-house media without cytokines to be cost effective in addition to providing high efficiency of differentiation in terms of characterization, in vitro functionality and large scale production of cells for therapeutic application. This study can further be enumerated with in vivo experiments in ischemic animal models thereby evaluating their safety and efficacy which can form the basis for further investigation.

\section{Conclusion}

Various researches have proved that several tissue-specific stem and progenitor cells exist in the hematopoietic system which has led to their isolation, delineation of, their properties and gene expression suggesting their potential application in regenerative medicine [18]. The observations from this study are:

$>$ CFU-ECs and ECFCs were detected in both the in-house media whereas commercial media produced only ECFCs.

$>$ Genotypic and phenotypic characterization showed that the cells derived in in-house media without addition of cytokines was comparable to the commercial media.

$>$ Functional assays involving taking up DiI-Ac-LDL and binding FITC-UEA-1 lectin were observed by EPCs cultured in both the in-house media like the commercial media.

$>$ EPCs cultured in all the media demonstrated homing potency by incorporating and integrating within the branching points and lengths of endothelial tubules. This property of endothelial cells was confirmed by using HFF which served as a negative control for tube assay.

$>$ High secretion of VEGF (46 pg/ml) and bFGF (10 pg/ml) was observed by EPCs cultured in media devoid of cytokines as compared to EPCs cultured in the other two media.

From this study, we conclude that $\mathrm{CD}^{+} 4^{+}$cells can be efficiently differentiated to functional EPCs. Thus, we have successfully derived a cost effective method of differentiating cells in enough numbers so that they can be clinically useful in required dosage thereby promising new hope for the treatment of various angiogenic disorders.

\section{Acknowledgement}

The authors acknowledge Reliance Life Sciences Pvt. Ltd (www.rellife.com) for providing the infrastructure and financial support to work on this project. The authors would also like to thank Dr. Shabari Sarang for providing CD34 ${ }^{+}$cells for the research.

\section{References}

1. Yoder MC (2012) Human endothelial progenitor cells. Cold Spring Harb Perspect Med 2: 1-18.

2. Ahrens I, Domeij H, Topcic D, Haviv I, Merivirta RM, et al. (2011) Successful In Vitro Expansion and Differentiation of Cord Blood Derived CD34 Cells into Early Endothelial Progenitor Cells Reveals Highly Differential Gene Expression. PLoS ONE 6: 1-12.

3. Murohara T, Ikeda H, Duan J, Shintani S, Sasaki Ki, et al. (2000) Transplanted cord blood-derived endothelial precursor cells augment postnatal neovascularization. J Clin Invest 105: 1527-36. 
4. Coultas L, Chawengsaksophak K, Rossant J (2005) Endothelial cells and VEGF in vascular development. Nature 438: 937-45.

5. Karamysheva AF (2008) Mechanisms of Angiogenesis. Biochemistry 73: 751-62.

6. Fan CL, Li Y, Gao PJ, Liu JJ, Zhang XJ, et al. (2003) Differentiation of endothelial progenitor cells from human umbilical cord blood CD34 ${ }^{+}$cells in vitro. Acta Pharmacol Sin 24: 212-8.

7. Asahara T, Murohara T, Sullivan A, Silver M, van der Zee R, et al. (1997) Isolation of putative progenitor endothelial cells for angiogenesis. Science 275: 964-7.

8. Case J, Mead LE, Bessler WK, Prater D, White HA, et al. (2007) Human CD34+AC133+VEGFR-2+ cells are not endothelial progenitor cells but distinct, primitive hematopoietic progenitors. Exp Hematol 35: 1109-18.

9. Ingram DA, Mead LE, Tanaka H, Meade V, Fenoglio A, et al. (2004) Identification of a novel hierarchy of endothelial progenitor cells using human peripheral and umbilical cord blood. Blood 104: 2752-60.

10. Tipnis S, Viswanathan C (2010) Umbilical Cord Matrix Derived Mesenchymal Stem Cells Can Change the Cord Blood Transplant Scenario. Int J Stem Cells 3: $103-18$.

11. Hristov M, Weber C (2004) Endothelial progenitor cells: characterization, pathophysiology, and possible clinical relevance. J Cell Mol Med 8: 498-508.

12. Zampetaki A, Kirton JP, Xu Q (2008) Vascular repair by endothelial progenitor cells. Cardiovasc Res 78: 413-21.

13. Shantsila E, Watson T, Lip GYH (2007) Endothelial Progenitor Cells in Cardiovascular Disorders. J Am Coll Cardiol 49: 741-52.

14. Yoder MC, Mead LE, Prater D, Krier TR, Mroueh KN, et al. (2007) Redefining endothelial progenitor cells via clonal analysis and hematopoietic stem/progenitor cell principals. Blood 109: 1801-9.

15. Voyta JC, Via DP, Butterfield CE, Zetter BR (1984) Identification and Isolation of Endothelial Cells Based on Their Increased Uptake of Acetylated-Low Density Lipoprotein. J Cell Biol 99: 2034-40.

16. Urbich C, Dimmeler S (2004) Endothelial Progenitor Cells: Characterization and Role in Vascular Biology. Circ Res 95: 343-53.

17. Folkman J (1995) Angiogenesis in cancer, vascular, rheumatoid and other disease. Nat Med 1:27-31.

18. Reya T, Morrison SJ, Clarke MF, Weissman IL (2001) Stem cells, cancer, and cancer stem cells. Nature 414: 105-11.

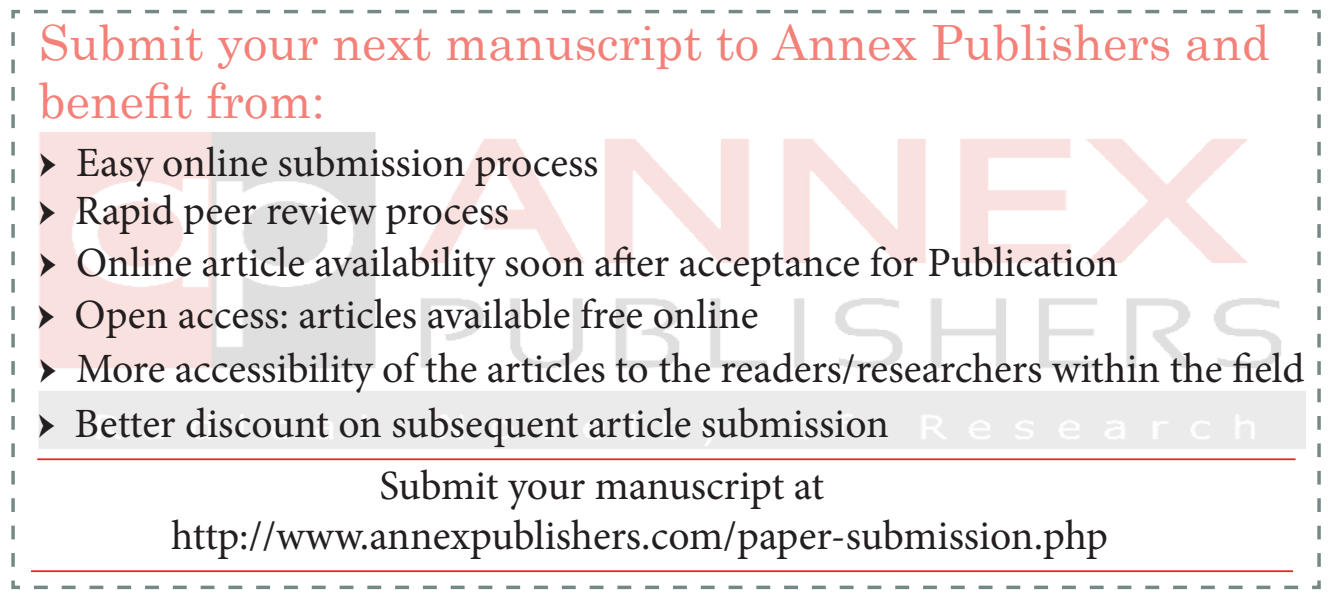

